\title{
Cerebellar Damage Loosens the Strategic Use of the Spatial Structure of the Search Space
}

\author{
Francesca Foti • Laura Mandolesi • Debora Cutuli • \\ Daniela Laricchiuta $\cdot$ Paola De Bartolo • \\ Francesca Gelfo • Laura Petrosini
}

(C) Springer Science + Business Media, LLC 2009

\begin{abstract}
The influence of a hemicerebellar lesion on the exploration of environments with different spatial distributions of multiple rewards was analyzed. Hemicerebellectomized (HCbed) and intact rats were submitted to a search task in which they had to explore nine food trays in an open field, avoiding repeated visits. Trays were spatially arranged in four configurations: cross, $3 \times 3$ matrix, circle, and three clusters of three trays each. Lesioned and intact rats' performances improved in all configurations used. However, the explorative activity of the HCbed animals differed from that of intact rats. Lesioned animals spent more time, made more errors, displayed lower search efficiency, exhibited shorter final spans, and traveled longer distances. They tended to perseverate and to neglect some trays. The cerebellar damage differentially influenced performances as a specific effect of the susceptibility of the configurations to being explored in a principled way. In the cross configuration that had strong spatial constraints, both groups made their lowest number of errors. In the circle configuration, the altered explorative strategies of lesioned animals made extremely demanding the acquisi-
\end{abstract}

F. Foti $\cdot$ L. Mandolesi $\cdot$ D. Cutuli $\cdot$ D. Laricchiuta $\cdot$ P. De Bartolo

F. Gelfo $\cdot$ L. Petrosini

IRCCS Santa Lucia Foundation,

Rome, Italy

F. Foti • D. Cutuli • D. Laricchiuta • P. De Bartolo •

L. Petrosini $(\bowtie)$

Department of Psychology, University "Sapienza" of Rome,

Via dei Marsi 78,

00185 Rome, Italy

e-mail: laura.petrosini@uniroma1.it

L. Mandolesi $\cdot$ F. Gelfo

University of Naples "Parthenope",

Naples, Italy tion of the task of searching multiple rewards, in spite of the attempt of favoring their altered procedures through an appropriate spatial arrangement. Since the procedural impairment elicited by cerebellar damage affected the central exploration, the matrix configuration was the most difficult configuration to be explored by the HCbed rats. The poor performances in the cluster configuration indicated that chunking was a strategy of relative strength in rats in general and in HCbed rats in particular.

Keywords Spatial arrangement · Explorative strategies · Spatial procedures $\cdot$ Clusters $\cdot$ Navigation $\cdot$ Rat

\section{Introduction}

One of the most interesting aspects of the cerebellar involvement in spatial function is its role in the explorative behavior. In fact, exploration requires close integration between environmental (sensory) information and explorative (motor) acts, thus mimicking the sensorimotor role classically attributed to cerebellar circuits. Recently, it was demonstrated that the cerebellar function in spatial event processing is primarily linked to the acquisition of procedural elements [1-4]. By using the Morris water maze (MWM), it has been demonstrated that hemicerebellectomized (HCbed) rats display a severe impairment in exploration strategies that are maintained even in the presence of a cerebellar lesion, if they are preoperatively learned $[1,2,5]$. Impaired performances are also displayed by HCbed animals in the radial arm maze (RAM), demonstrating that cerebellar damage induces an inflexible use of procedures (if indeed any procedure can be acquired in the presence of a cerebellar lesion) and a severe impairment in working memory processes [3]. Furthermore, in the presence of a 
cerebellar lesion, it is possible to detect the environmental changes only when the altered procedural abilities were favored by environmental arrangement [6]. Data obtained in mutant mice support the cerebellar involvement in spatial learning in general [7-9] and in the explorative behavior in particular [10-16].

In the lab settings, innate foraging behaviors, learning of searching strategies, motivation to explore, and modification of explorative behaviors in the presence of brain injury or pharmacological treatments have been studied by using tests of activity (RAM, MWM, open field with or without objects) with constrained or unconstrained trajectories and with single or multiple rewards. In the RAM, the searching strategies to reach the multiple rewards inevitably are forced by the intrinsic structure of the apparatus that, by itself, allows algorithmic strategies, as for example visiting adjacent arms in succession, which minimize the mnesic task demands. Conversely, in the unconstrained tests of exploration, such as MWM or open field, a lot of trajectories from the starting point to the target can be traveled over.

When multiple rewards are distributed in unconstrained environments, the exploratory behaviors have to adapt to the environment features so that spatial structure of the search space influences the economy and organization of searching behaviors. The immediate background of the present experiment is a previous study [17] on intact naïve rats submitted to a search task in which the animals had to explore an open field with multiple food trays arranged according to different spatial configurations, without visiting already-depleted trays. Rats exhibited differential search efficiency as a specific effect of the susceptibility of the configurations to being explored in a principled way.

Given that structural affordances of the environment influence the construction of search strategies as well as the information on where the reward is, we wondered whether and how the procedural impairment elicited by a cerebellar lesion affected search strategies to explore different spatial configurations of multiple rewards. In particular, the aim of our study was to analyze whether the presence of cerebellar lesion prevented either remembering visited locations or organizing principled search patterns and thus did not allow an efficient exploration or whether vice versa the procedural impairment of cerebellar origin did not result in disorganized search patterns. Furthermore, we analyzed whether the cerebellar lesion impaired the use of chunking abilities as a means of reducing data in a task that made demands on the memory system.

Hemicerebellectomy $(\mathrm{HCb})$ was chosen as the experimental model of the cerebellar lesion because it provoked less disrupting motor effects than a complete cerebellectomy [18], and thus it allowed locomotor performances (exploration of the arena), as required by the present task.
Furthermore, it has been repeatedly demonstrated that in rats even a unilateral cerebellar lesion is able to affect a large range of cognitive functions [1, 3, 6, 19-24].

In the present task, the animals had to explore an open arena to search a set of nine food trays arranged according to four spatial configurations. The animals had to keep track of the visits made over time in the absence of any constrained trajectory to travel and of physical traces left from choices they had already made. The spatial configurations were a cross, a $3 \times 3$ matrix, three clusters of three trays each, and a circle [17, 25-27]. Such configurations allowed analyzing how the spatial structure of the search space could affect the search patterns of HCbed animals.

\section{Experimental Procedures}

\section{Subjects}

Forty adult male Wistar rats were used in the present research. At the beginning of experiments, the mean age of animals was 3.6 months \pm 14 days. The animals were housed two animals to a cage $(42 \times 26 \times 18 \mathrm{~cm})$ furnished with wood shaving bedding and kept on a standardized dark/light schedule (12/12 h; lights on at 0700 hours). The room temperature was $21^{\circ} \mathrm{C}$ and the relative humidity $60 \pm$ $5 \%$. All experiments were carried out according to the European Community Council Directives of November 24, 1986 (86/609/EEC).

\section{Experimental Groups}

The animals used in the present research were randomly assigned to one of two experimental groups: control group $(n=20)$, comprising intact naïve animals, and HCbed group $(n=20)$. Since no definite indication is present in the literature as for any behavioral lateralization of cerebellar structures [22], in all lesioned animals, the unilateral cerebellar lesion was performed on the right side, on the analogy of previous studies $[1,3,6,19-21,23,24]$. The animals of each main experimental group were subsequently randomly assigned to one of the four different subgroups, each of which included five animals, according to the different spatial tray arrangement.

\section{Surgery and Motor Assessment}

Rats were anesthetized with Zoletil 100 (tiletamine and zolazepam $50 \mathrm{mg} / \mathrm{kg}$ i.p.-Virbac s.r.l., Milan, Italy) and Rompun (xylazine $10 \mathrm{mg} / \mathrm{kg}$ i.p.-Bayer s.p.a., Milan, Italy). A craniotomy was performed over the right hemicerebellum. The dura was excised, and the right cerebellar hemisphere and hemivermis were ablated by suction. Care 
was taken not to lesion extracerebellar structures. The cavity was filled with sterile gel foam; the wound edges were sutured, and the animals were allowed to recover from anesthesia and surgical stress. Testing was performed 3 weeks after $\mathrm{HCb}$, when no change in cerebellar symptomatology was observed. Animals were submitted to behavioral testing only if they exhibited stable motor symptomatology consistent with a cerebellar lesion. Details and time course of the cerebellar symptoms in the rats are described elsewhere [18]. The HCbed rats displayed extensor hypotonia ipsilateral to the lesion, resulting in an asymmetrically crouched posture with body tilt to the right. In motion, the HCbed rats tended to collapse on their bellies and their wide-based gait was slightly ataxic. In spite of the motor symptomatology, the HCbed animals did not exhibit such severe akinetic symptoms as to impede reliable testing.

\section{Apparatus}

The apparatus was placed in a lab that was dimly and uniformly illuminated by a masked neon ceiling lamp. It consisted of a round plywood table $(150 \mathrm{~cm}$ in diameter, $2.5 \mathrm{~cm}$ thick) raised $50 \mathrm{~cm}$ from the floor by a rotating support. There was a $50-\mathrm{cm}$ gray opaque wall around the table. The wall impeded accidental falls possible mainly in HCbed rats and greatly reduced the number of external cues in the arena. However, use of spatial cues from the ceiling was not prevented because there was no cover over the arena. Nine blue chemically inert tube caps $(3 \mathrm{~cm}$ in diameter, $2 \mathrm{~cm}$ deep) used as food trays were arranged according to the spatial configurations described in the "Procedures" section. The depth of the tray prevented the rats from seeing the reward at a distance but allowed for an easy reward, i.e., eating. The reward was a single piece of the standard food for rats (Mucedola 4RS21 standard diet GLP complete feed for mice and rats) sweetened by condensed milk (Nestlé Italiana, Milan, Italy).

\section{Pretraining}

Three days before the testing, the animals were placed on food deprivation with free access to water. They were reduced to $90 \%$ of ad lib weight by scheduled feeding (30 min of free feeding every day) and were maintained at this level throughout the experiment by being given a restricted amount of the standard food each day. The animals were weighed once a day. The food deprivation lasted 8 days. During this period, the rats showed no signs of aggression during or between feeding times or signs of distress. The rats' behavior in their cages was monitored twice a day. At the end of testing, no significant difference was found in the body weight of the animals in the experimental groups (mean values $( \pm \mathrm{SD})$ recorded on the last day of testing: C group $464 \pm 44 \mathrm{~g}$; HCbed group $459 \pm 38 \mathrm{~g}$ ).

Before the experiment began, the rats were submitted to 3-day pretraining. On the first day, the animals were manipulated for $10 \mathrm{~min}$ in their home cage, which had been placed in the experimental lab, to become accustomed to the experimenters and the lab environment. On the second day, pairs of animals were allowed to explore the table freely for $15 \mathrm{~min}$, and three baited food trays were randomly arranged on the table. On the last day of pretraining, individual rats were again placed on the table with three baited trays and allowed to explore it for $15 \mathrm{~min}$. During the second and third days of pretraining, the animals visited the food trays and emptied their contents during the 15 min of exploration. Testing sessions began the next day.

\section{Procedures}

Spatial configurations were derived from preceding studies [17, 25-27] (Fig. 1). In the circle configuration, the trays were arranged to form a circumference (diameter $80 \mathrm{~cm}$ ) with trays $25 \mathrm{~cm}$ apart. In the matrix configuration, the food trays were arranged in a $3 \times 3$ square matrix in the center of the table, with trays $25 \mathrm{~cm}$ apart. In the cross configuration, the trays were arranged in an " $\mathrm{X}$ " formation in the center of the table, with trays $25 \mathrm{~cm}$ apart. In the cluster configuration, the trays were arranged in triplets $120^{\circ}$ away from each other, with the center of each triplet placed $40 \mathrm{~cm}$ away from the center of the table and trays placed $25 \mathrm{~cm}$ apart.

In each trial, the goal was to collect all nine rewards, which were never replaced during the trials. At the end of

Fig. 1. Tray arrangement in the four configurations (circle, matrix, cross, cluster). The starting points in circle, matrix, and cross configurations are defined by the cardinal points $(\mathrm{N}, \mathrm{S}, \mathrm{W}$, or $\mathrm{E})$, and the starting points in cluster configuration $(\mathrm{N}, \mathrm{SW}$, or SE) are indicated

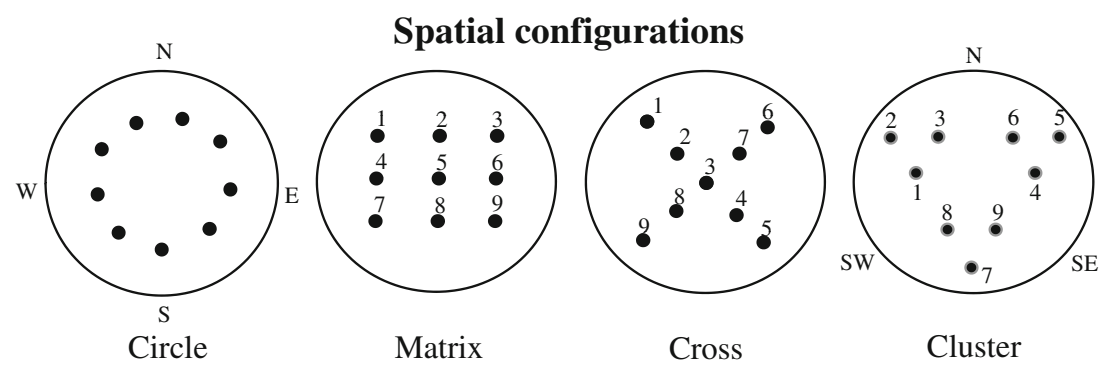


each trial, the rats were put back in their cages for $15 \mathrm{~min}$. During this period, the table was cleaned but the trays were not and a new piece of food was added in each tray. Each rat underwent six trials per session, one session a day for five consecutive days. The rats' trajectories in the arena were monitored by a video camera mounted on the ceiling. The resulting video signal was relayed to a monitor and to an image analyzer (Ethovision, Noldus, Wageningen, The Netherlands).

At the beginning of the trial, the rat was placed on the table facing the center at one of the starting points defined by the cardinal points $(\mathrm{N}, \mathrm{S}, \mathrm{W}$, or E) in cross, matrix, and circle configurations and at one of three starting points $(\mathrm{N}$, SW, or SE) in the cluster configuration (Fig. 1). Starting points were balanced across trials. To further reduce spatial encoding of the food source position based on external cues, at the beginning of each trial, the table was rotated to one of four different positions $(\mathrm{N}, \mathrm{S}, \mathrm{W}$, or $\mathrm{E})$ in a counterbalanced order.

In each configuration, we considered the position of the single trays, subdividing them in external (matrix 12346 78 9; cross 1569 ; cluster 257 ) and internal (matrix 5; cross 234478 ; cluster $1346 \quad 8$ 9) trays. Such a subdivision of trays was not applied in the circle configuration where all trays were localized at the same distance from the arena center.

To analyze whether the reward odor from the food trays could guide search behavior, a nonreinforced probe trial was performed as the seventh trial after the six trials of the last session of the circle configuration. In this probe trial, food trays were carefully washed and left empty.

\section{Behavioral Parameters}

Rats were allowed to freely explore the apparatus. A trial ended when all nine rewards had been collected or 30 choices had been made or $15 \mathrm{~min}$ had elapsed. A visit was defined as nose poking or touching a food tray to sniff it. Since the food trays were never rebaited, the best performance consisted of visiting all nine trays only once.

In each of the six trials of a session, the following parameters were analyzed: search time, the time (in seconds) employed to complete the trial; visits, performed to each tray in a trial; percentage of total errors performed in a trial (considering either revisits and no visits to a tray so that it remained baited); no visits, considered as ignoring one or more trays without visiting it during the entire trial; search efficiency, the percentage of correct visits out of total visits; final span, defined as the longest sequence of correct visits reached in the last session; perseverations, considered as the sum of times in which the animal consecutively revisited the same trays in each trial (the tray revisited could be either the same tray, i.e., 5-5, or a sequence of maximum three trays, i.e., 1-2-3-1-2-3). Perseverations on more than three trays were never observed. Also, the distance (in centimeter) traveled on the table and the distance traveled in exploring a peripheral annulus with a $20-\mathrm{cm}$ radius were calculated. The trajectories drawn by the image analyzer were also considered. The kind of trajectory, direct, or indirect, used to reach a tray, either correct or incorrect, was also analyzed. The trajectory was considered direct when the distance traveled by the animals to reach a new tray exceeded the real between-tray distance by no more than $10 \%$ when the trajectories of control animals were analyzed. Since HCbed rats exhibited a tendency to perform curvilinear paths, their trajectories were considered direct when the traveled distance did not exceed the real between-tray distance by $30 \%[6,21]$.

In the cluster configuration, the number of clusters visited or revisited in each trial was analyzed (this parameter ranged from the worst value of 30 to the best value of 3). Furthermore, the errors were classified as within-cluster errors, that is the revisits to a tray belonging to the same cluster the rat was visiting, across-cluster errors, that is the late revisits to an incorrect tray belonging to a previously visited cluster.

To verify the anxiety levels, some emotional parameters were analyzed in both experimental groups. To this aim, the number of defecation boluses and motionless time was recorded in each trial.

\section{Histological Controls}

When the behavioral testing was finished, the HCbed animals were deeply anesthetized and transcardially perfused with saline followed by $4 \%$ buffered formalin. The extent of the cerebellar lesion was determined from Nisslstained $50-\mu \mathrm{m}$ frozen sections. Animals were included in the present study if they had received a complete right $\mathrm{HCb}$ with total ablation of deep nuclei (Fig. 2). In all cases reported here, the left side of the cerebellum and all extracerebellar structures were completely spared, except for the dorsal cap of the right Deiters' nucleus which in some cases was slightly affected. The variability in the extent of the floccular and vermian lesions was considered not influencing because in all cases these structures were functionally disconnected due to the ablation of the cerebellar peduncles and deep nuclei of the right side.

\section{Statistical Analysis}

Metric unit results of animals belonging to the experimental groups (presented as mean values of the six trials in one session (day) for individual animals \pm SEM) were first tested for homoscedasticity by means of Levene test and for normality by means of Shapiro-Wilk normality test. The 


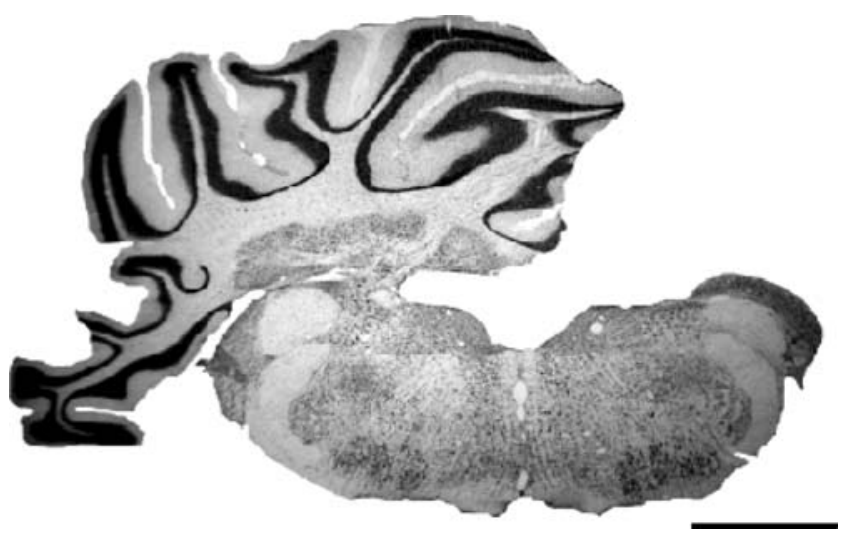

Fig. 2. Nissl-stained coronal section through cerebellum and brain stem in a HCbed rat. Note the total absence of the right hemicerebellum and the sparing of any extracerebellar structure. Scale bar $2 \mathrm{~mm}$

values were then compared using one-way, two-way (with group as between-subject factor and session (or error) as within-subject factor), or three-way (with group as between-subject factor and trajectory and correctness as within-subject factors) analyses of variance (ANOVAs), followed by multiple comparisons using Duncan's test. Correlation between data was also tested by means of Pearson's $r$. The $p$ level considered as statistical significance was $p<0.05$.

\section{Results}

Emotional Parameters Control and HCbed animals exhibited comparable levels of anxiety. In fact, in the main groups of HCbed and control animals, defecation boluses were produced only in the very first sessions, independent from the spatial arrangement of trays. No significant differences between groups were found $\left(F_{1}, 38=2.17\right.$, $p$ ns). The other parameter indicative of a high-stress state is a freezing or motionless period. Since none of the animals exhibited this behavior, even in the very first trials, no statistical comparison was made.

Search Time In all configurations, the HCbed animals spent more time to complete the task in comparison to control animals, although in the circle and matrix configurations, the between-group difference failed to reach significance levels (Table 1; Fig. 3a).

Visits In all configurations, the external and internal trays were equally visited by control animals (one-way ANOVAs: cross: $F_{1,4}=1.63, p$ ns; cluster: $F_{1,4}=0.95$, $p$ ns; matrix: $F_{1,4}=0.07, p$ ns). Conversely, the HCbed rats exhibited a reduced visiting of the internal tray/trays in matrix and cluster configuration (cross: $F_{1,4}=0.27 ; p$ ns; matrix: $F_{1,4}=7.8 ; p=0.05$; cluster: $F_{1,4}=7.7 ; p=0.05$; Fig. 3b, c).

Total Errors In all configurations, the HCbed animals performed a significantly higher number of errors in comparison to control animals. However, total errors progressively diminished as the sessions went by in both experimental groups (Table 1; Fig. 4a). While control animals exhibited a mean value of 6.29 errors in the matrix, 5.93 errors in the cluster, 5.14 errors in the circle, and 3.89 errors in the cross configuration, the HCbed animals performed 9.17 errors in the matrix, 9.03 errors in the circle, 7.69 errors in the cluster, and 7.51 errors in the cross configuration, exhibiting thus higher values of errors in all configurations.

No Visits One of the parameters that mostly differentiated the two main groups was the occurrence of no visits, i.e., neglecting one or more trays without visiting it during the entire trial. Interestingly, while control animals exhibited a number of no visits very close to zero in all configurations since the first session, HCbed animals neglected up to four trays in the cluster and matrix configurations in the first session (cluster 4.57; matrix 4.21; circle 1.95; cross 1.64) and reached values near to zero only in the last session (Table 1; Fig. 4b).

Search Efficiency The rats never attained the most efficient performance in absolute terms because in any configuration the number of visits to exhaustively conclude the trial was never near nine, i.e., a value that indicated $90-100 \%$ search efficiency. Namely, control animals reached their best search efficiency value $(83 \%)$ corresponding to $11.8 \pm 0.8$ visits in the circle configuration, while HCbed animals reached their best value $(67 \%)$ corresponding to $14.3 \pm 0.7$ visits in the cluster configuration (Table 1; Fig. 4c).

Final Span The longest sequence of correct visits was exhibited by control animals in the circle configuration $(\bar{x}: 7.1 \pm 1.0)$, while HCbed rats exhibited their longest sequence in matrix configuration $(\bar{x}: 5.60 \pm 1.17$; Table 1 ; Fig. 5a).

Perseverations A clear between-group difference was found given that HCbed animals displayed a high number of perseverations in almost all configurations. Interestingly, as shown in Fig. 5b, both experimental groups displayed the absence of perseverations in the cross configuration (Table 1). While control animals exhibited some perseverations in the first session (circle 4.4 \pm 0.74 ; matrix 9.6 \pm 3.14 ; cluster $4.8 \pm 1.62$ ) but reached values near to 1 in the last session in any configuration, HCbed animals performed 
Table 1 Statistical comparisons (two-way ANOVAs) of behavioral responses in the circle, matrix, cross, and cluster configurations

\begin{tabular}{|c|c|c|c|c|c|c|c|}
\hline \multirow[t]{2}{*}{ Arrangements } & \multirow[t]{2}{*}{ Parameters } & \multicolumn{2}{|l|}{ Group effect } & \multicolumn{2}{|l|}{ Session effect } & \multicolumn{2}{|l|}{ Interaction } \\
\hline & & $F$ value $(d f=1,8)$ & $p$ & $F$ value $(d f=4,32)$ & $p$ & $F$ value $(d f=4,32)$ & $p$ \\
\hline \multirow[t]{8}{*}{ Circle } & Search time & 1.70 & ns & 7.72 & 0.0001 & 0.27 & ns \\
\hline & Total errors & 6.32 & 0.03 & 6.96 & 0.0003 & 0.90 & ns \\
\hline & No visits & 1.90 & ns & 0.59 & 0.5 & 2.15 & ns \\
\hline & Search efficiency & 6.93 & 0.03 & 6.46 & 0.001 & 1.08 & ns \\
\hline & Final span & 4.06 & ns & & & & \\
\hline & Perseverations & 5.79 & 0.04 & 3.24 & 0.02 & 1.23 & ns \\
\hline & Total distance & 1.86 & ns & 5.80 & 0.001 & 0.95 & ns \\
\hline & Peripheral distance & 1.44 & ns & 6.13 & 0.001 & 1.09 & ns \\
\hline \multirow[t]{8}{*}{ Matrix } & Search time & 2.98 & ns & 20.25 & 0.00001 & 2.73 & 0.04 \\
\hline & Total errors & 5.44 & 0.04 & 15.02 & 0.00001 & 0.43 & ns \\
\hline & No visits & 17.52 & 0.003 & 8.62 & 0.00007 & 4.82 & 0.003 \\
\hline & Search efficiency & 4.36 & ns & 12.85 & 0.00001 & 0.87 & ns \\
\hline & Final span & 0.64 & ns & & & & \\
\hline & Perseverations & 5.71 & 0.04 & 22.18 & 0.00001 & 0.70 & ns \\
\hline & Total distance & 0.45 & ns & 22.59 & 0.00001 & 1.92 & ns \\
\hline & Peripheral distance & 0.41 & ns & 18.60 & 0.00001 & 1.53 & ns \\
\hline \multirow[t]{8}{*}{ Cross } & Search time & 9.07 & 0.01 & 2.31 & ns & 0.94 & ns \\
\hline & Total errors & 117.72 & 0.00001 & 4.10 & 0.008 & 0.72 & ns \\
\hline & No visits & 1.49 & ns & 0.83 & ns & 0.88 & ns \\
\hline & Search efficiency & 66.09 & 0.00001 & 4.73 & 0.004 & 0.36 & ns \\
\hline & Final span & 13.80 & 0.01 & & & & \\
\hline & Perseverations & - & - & - & - & - & - \\
\hline & Total distance & 33.52 & 0.001 & 5.68 & 0.001 & 2.20 & ns \\
\hline & Peripheral distance & 216.69 & 0.00001 & 6.84 & 0.005 & 3.80 & 0.01 \\
\hline \multirow[t]{8}{*}{ Cluster } & Search time & 30.69 & 0.0005 & 8.73 & 0.0001 & 2.16 & ns \\
\hline & Total errors & 24.27 & 0.001 & 17.81 & 0.0001 & 0.38 & ns \\
\hline & No visits & 12.34 & 0.007 & 1.60 & ns & 1.30 & ns \\
\hline & Search efficiency & 11.78 & 0.01 & 20.15 & 0.0001 & 0.77 & ns \\
\hline & Final span & 6.95 & 0.02 & & & & \\
\hline & Perseverations & 9.91 & 0.01 & 12.02 & 0.00001 & 2.48 & ns \\
\hline & Total distance & 19.57 & 0.01 & 12.01 & 0.00001 & 1.69 & ns \\
\hline & Peripheral distance & 7.22 & 0.05 & 16.43 & 0.00001 & 1.18 & ns \\
\hline
\end{tabular}

numerous perseverative errors in the first session (circle $15.4 \pm 6.0$; matrix $14.2 \pm 1.01$; cluster $12.0 \pm 1.54$ ) and still persevered in the last session (circle 7.4 \pm 2.97 ; matrix $3.4 \pm$ 0.81 ; cluster $3.8 \pm 1.24$ ).

Traveling Distances Both total and peripheral distances traveled in foraging the arena were significantly influenced by the cerebellar lesion in cluster and cross configurations where lesioned animals traveled longer distances in comparison to controls (Table 1). In spite of it, both total and peripheral distances were reduced as the sessions went by in all configurations in both experimental groups (Fig. 5c).
Explorative Rules To detail the explorative rules applied in picking up the rewards, we analyzed the kind of trajectory (direct or indirect) used in reaching a new tray by distinguishing whether the tray was correct or incorrect.

As shown in Fig. 6, in all configurations, control animals made the most visits to correct trays through direct trajectories (mean percentage 59.0\%), whereas their least frequent response was a visit to a correct tray through an indirect trajectory. Incorrect trays were most frequently reached through indirect trajectories in cluster and least frequently in circle configurations. Interestingly, this pattern was opposite to that found when incorrect trays were reached through direct trajectories. 

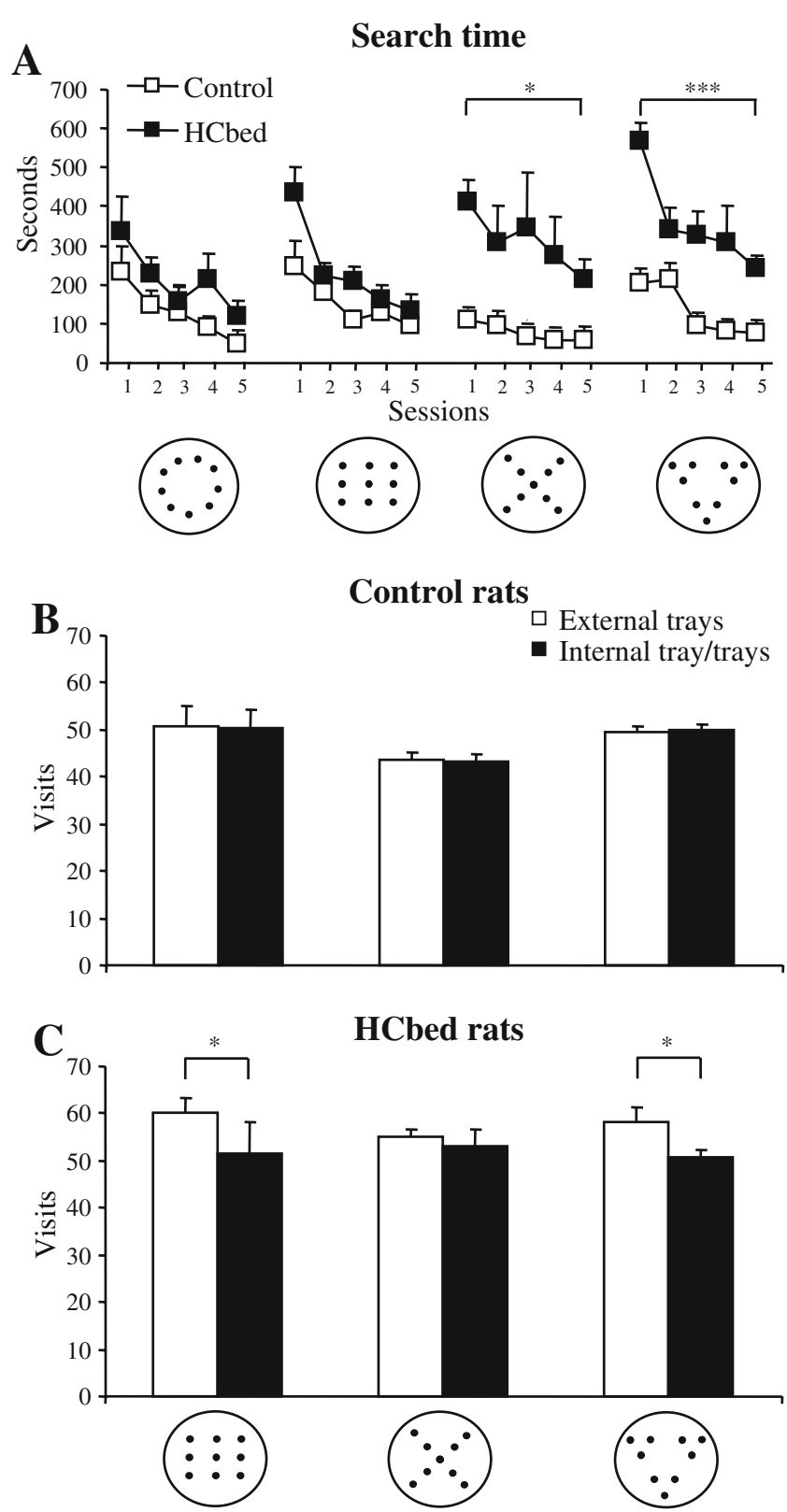

Fig. 3. Performances displayed by HCbed and control rats across the five sessions of the search task in circle, matrix, cross, and cluster configurations. a Mean ( \pm SEM) search time used to complete the task. b, c Mean number ( \pm SEM) of visits to external and internal trays made by control (b) and HCbed (c) rats in matrix, cross, and cluster configurations. In this and in the following figure, the asterisks indicate the significance level of the main factor "group" of the respective two-way analyses of variance $\left({ }^{*} p \leq 0.05 ; *^{*} p \leq 0.001\right.$; $* * * p \leq 0.0005 ; * * * * p \leq 0.0001)$

Even HCbed animals made the most visits to correct trays through direct trajectories; however, no significant differences among the four configurations were observed (mean percentage 39.2\%). Also, in HCbed animals, the least frequent response was a visit to a correct tray through an indirect trajectory, but the percentages of this pattern were significantly higher than those observed in control
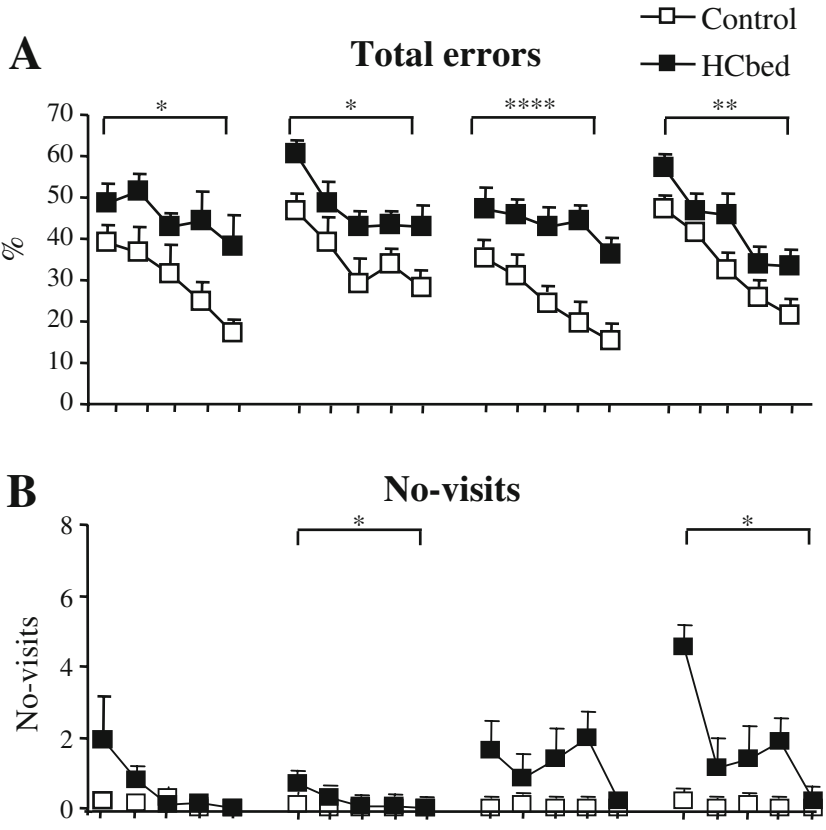

C

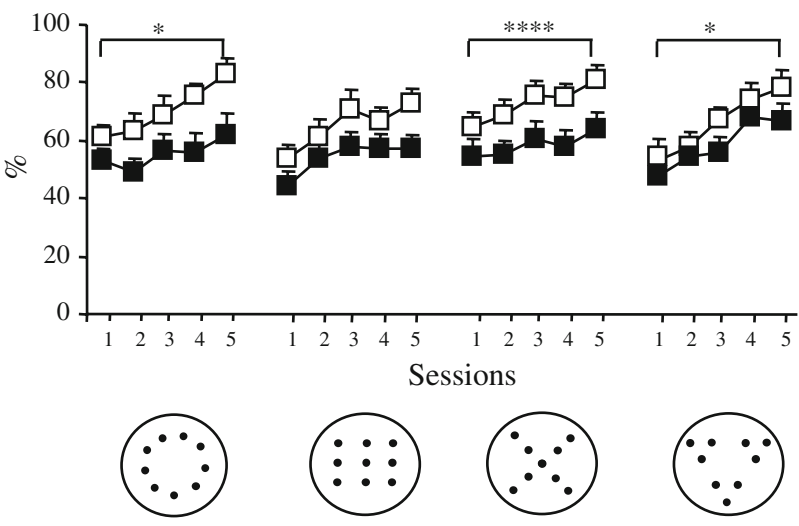

Fig. 4. Performances displayed by HCbed and control rats across the five sessions of the search task in circle, matrix, cross, and cluster configurations. a Mean $( \pm$ SEM) total errors. b Mean $( \pm$ SEM $)$ no visits. c Mean $( \pm$ SEM) search efficiency (percentage of correct visits on the total visits)

animals. When the incorrect trays were reached through indirect trajectories, no differences among configurations were observed. Finally, incorrect trays were most frequently reached through direct trajectories in the matrix and least frequently in the cross configurations.

\section{Search Strategies in Cluster Configuration}

In a configuration displaying closely grouped rewards, such as the cluster configuration, rats should complete each cluster before moving on to the next one. In this way, they have only three items to remember at a time (the three trays within a cluster) and the position of the three clusters. 
A
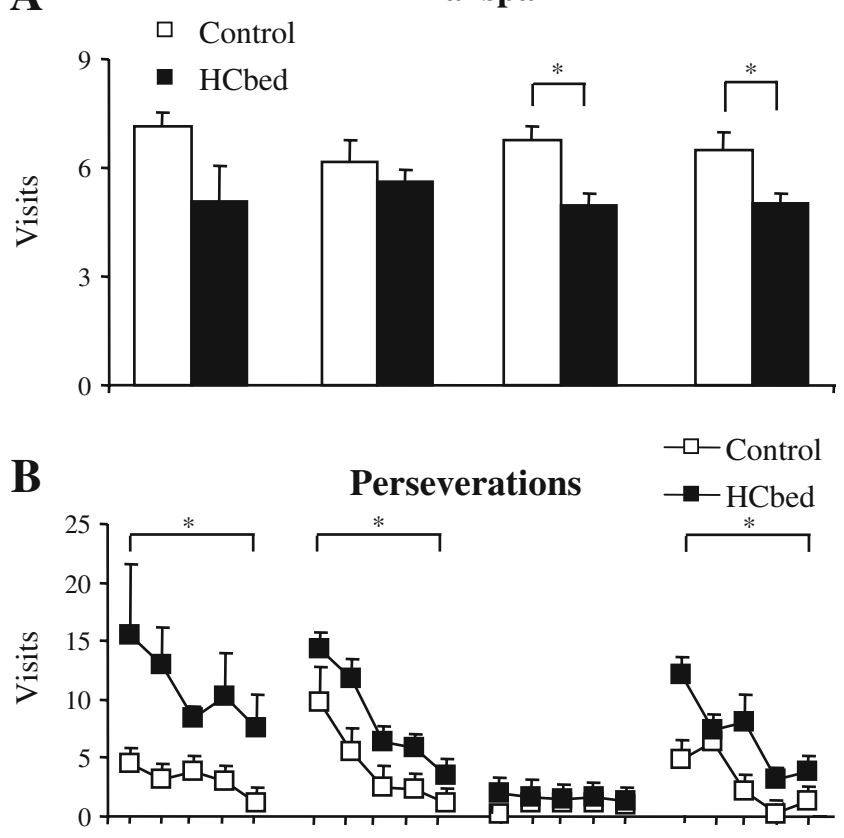

C

Total distance

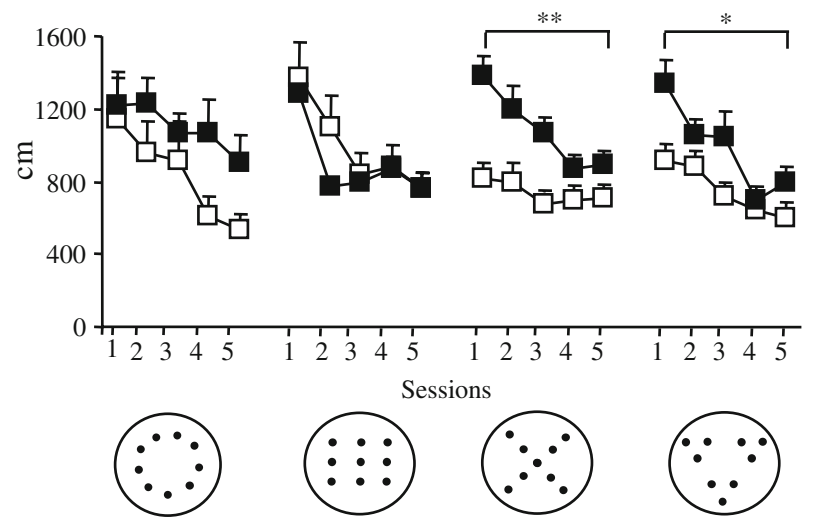

Fig. 5. Performances displayed by HCbed and control rats in the search task in circle, matrix, cross, and cluster configurations. a Mean ( \pm SEM) final span (longest sequence of correct visits) displayed by the animals in the last session of the task. b Mean $( \pm$ SEM) perseverative errors exhibited by the animals across the five sessions of the search task. c Mean ( \pm SEM) total distances traveled across the five sessions of the search task

Additional parameters were considered to analyze whether the rats used the spatial constraints afforded by the clustered space in this principled manner. A two-way ANOVA (group $\times$ session) revealed a significant session effect in the number of clusters visited $\left(F_{4,32}=14.14 ; p<\right.$ $0.00001)$, while lesion $\left(F_{1,8}=0.07 ; p \mathrm{~ns}\right)$ and interaction $\left(F_{4,32}=0.50 ; p \mathrm{~ns}\right)$ were not significant. We also analyzed whether the kind of errors (within cluster and across cluster) the animals made was significantly influenced by the presence of the lesion. A two-way ANOVA (group $x$ error) revealed a significant effect of the kind of error $\left(F_{2,16}=3.75 ; p=0.045\right)$ and a not significant group $\left(F_{1,8}=\right.$

\section{Explorative rules}

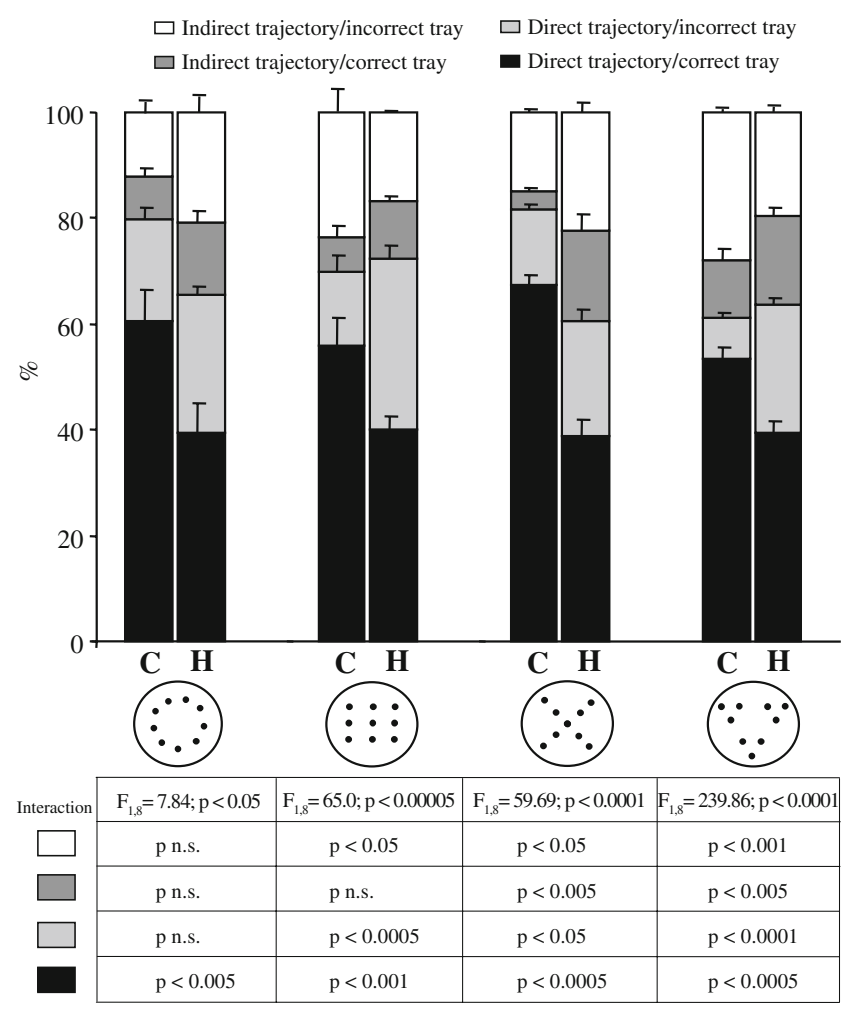

Fig. 6. Performances displayed by HCbed $(H)$ and control $(C)$ rats in the search task in circle, matrix, cross, and cluster configurations. The mean $( \pm$ SEM) percentages of correct (or incorrect) trays visited through direct (or indirect) trajectories are depicted. In the table, the second-order interaction of the respective three-way ANOVAs (group $\times$ trajectory $\times$ correctness) as well as the levels of significance of the post hoc comparisons between groups in the four trajectory/ correctness combinations is indicated

$3.19 ; p$ ns) effect. The interaction was also significant $\left(F_{2,16}=3.65 ; p=0.047\right)$. Post hoc comparisons revealed that HCbed animals performed a significantly $(p=0.04)$ higher number of within-cluster errors in comparison to control animals.

The existence of a relationship between the use of principled search and the ability to remember the already-visited sites was further supported by the analysis of the relationship between the number of across-cluster errors and the number of clusters visited. While in the control animals a high parallelism between measures emerged (Pearson's $r=0.98$ ), in the HCbed animals the measures appeared much less correlated (Pearson's $r=0.48$ ). This finding indicates that control animals made more errors when they visited more clusters because abandoning a cluster resulted in visiting already-depleted trays when the cluster was later revisited. Conversely, the HCbed animals exhibited the low correlation between measures because they tended to revisit already-depleted trays belonging to the cluster they were exploring, as demonstrated by their high number of within-cluster errors. 


\section{Probe Trial}

In the circle configuration, the number of errors made in a nonreinforced probe trial (C: $\bar{x}=3.4 \pm 2.2$; HCbed: $\bar{x}=8.0 \pm 3.8)$ was compared with the number of errors made in the sixth trial of the last session $(\mathrm{C}: \bar{x}=3.2 \pm 3.2$; HCbed: $\bar{x}=8.8 \pm 2.3$ ). One-way ANOVAs showed no significant effect in both experimental groups $\left(\mathrm{C}: F_{1,8}=\right.$ $0.002 ; p$ ns; HCbed: $F_{1,}=0.03 ; p$ ns). This finding indicated that performances were comparable in reinforced and nonreinforced trials, thus excluding that the odor traces of the reward guided the rats to baited trays or away from depleted ones.

\section{Discussion}

The present study investigated the influence of a cerebellar lesion on rat exploratory activity in environments featured by different spatial distributions of multiple rewards. The presence of rewards allowed maintaining high levels of motivation. The animals not only did not diminish exploration (habituation to the environment) [6], but they learned the reward positions, displaying progressively more tuned searching behaviors. Thus, as the sessions went by, lesioned and intact rats' performances improved in all configurations used and for almost all parameters considered. However, in spite of the presence of learning in all animals, the exploratory activity of the HCbed animals significantly differed from that displayed by intact rats (Fig. 7). Namely, in exploring the different spatial configurations, in comparison to intact animals, HCbed rats spent more time, made more errors, displayed lower search efficiency, exhibited shorter final spans, and traveled longer total and peripheral distances. Furthermore, at odds with intact animals, the lesioned rats tended both to perseverate (consecutively revisiting the same trays) and to neglect some trays ("skipping" them in the succession of visited trays), both indices of disorganized explorative functions. Interestingly, the most efficient explorative rule (represented by reaching a not-visited tray through a direct trajectory) was applied less frequently by the lesioned animals than by intact animals, although it was the strategy most used by all experimental groups.

Given the reduced presence of extramaze (allothetic) cues that were available only from ceiling, intramaze and idiothetic cues were used to learn the task. The intramaze cues were derived from the spatial relationships specified
Fig. 7. Explorative trajectories of HCbed and control rats in exploring the circle, matrix, cross, and cluster configurations. The trajectories traveled by all animals of each group in the first and last trial of the task are reported

\section{Explorative trajectories}

Control

HCbed

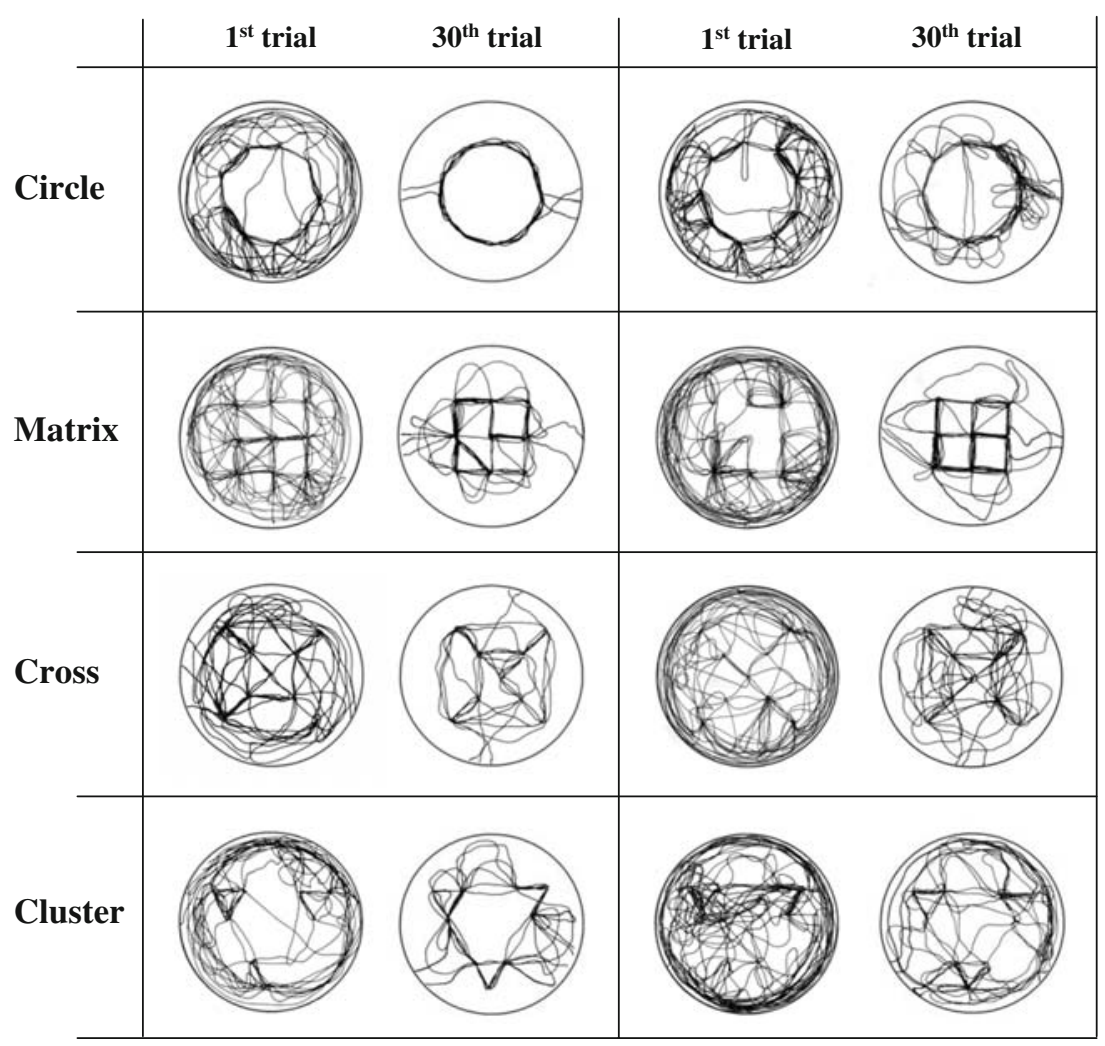


by the trays themselves. The idiothetic cues were derived from the rats' own movements. They encompassed internal self-motion information provided by the vestibular, proprioceptive, and somatosensory systems; efference copies of motor commands; and external motion-related information such as optic flow [28-32]. The present findings on the defective explorative activity of the HCbed animals are in agreement with the general framework that considers the cerebellar structures heavily involved in processing motor performance-related signals [33]. In fact, in the knowledge of motion through space, the cerebellar circuits are implicated in acquiring information necessary for spatial orientation, self-motion perception, and postural control [34-36].

Once the contribution of cerebellar networks in the explorative behaviors is ascertained, let us analyze whether the different spatial distributions of the rewards affected the HCbed rats' search behavior.

\section{Exploration of Cross Configuration}

In the cross configuration that had strong spatial constraints, both control and HCbed rats depleted their highest number of trays, making their respective lowest number of errors. To fully deplete the cross, the rats could use an endto-end search pattern twice, moving along the lines and visiting adjacent trays. The trajectory adopted to explore a cross could work as a notational system that aided the use of a principled pattern and thus strongly reduced the memory load. This characteristic of the cross configuration allowed a rather efficient exploration and prevented the occurrence of perseverative errors even in the presence of cerebellar damage. However, to exhaustively deplete trays located in straight lines, a firm directional principle preventing the inversion of the travel direction should be required. Conversely, more frequently than the control animals, the HCbed rats displayed abrupt travel inversions that provoked revisits. This disorganized navigational strategy lowered their search efficiency to the relatively good value of $64 \%$.

\section{Exploration of Circle Configuration}

Also, the circle configuration could be used as a notational system that reduced the amount of information the subjects had to memorize to avoid repeated visits. Once more, a subject always moving to the adjacent loci would not need to memorize the locations already visited because the particular point of the circle the animal was in told it unambiguously the trays already visited and those to be visited. Thus, this was a very robust strategy because the trajectory itself acted as a notational system. As a further notation, it has to be noted that trays were placed to a distance great enough from the wall, so that the animals displaying thigmotaxic tendencies accidentally would not bump into objects and, on the other hand, sufficiently far from arena walls to require active exploration. This distance and the lack of a central tray did not demand the exploration of the inner areas of the arena in the circle configuration, fostering the biased explorative strategies of HCbed animals that as a rule tended to explore the peripheral sectors of the arena. We retained that a spatial configuration shaped up according to the explorative tendencies of the HCbed animals could favor their learning. Conversely, the circle configuration was not the configuration most efficiently explored by the HCbed animals. In fact, in exploring it, the cerebellar rats displayed a lot of total errors and perseverative errors, search efficiency not reaching their maximal value and low final span. HCbed animals did not exploit the circular arrangement of the rewards because they put into action abrupt inversions of travel direction retracing their own steps. This unbefitting strategy caused errors and in some cases even perseverative errors. These findings indicate that altered explorative strategies made extremely demanding the acquisition of the task of searching multiple rewards, in spite of the attempt of favoring their altered procedures through an appropriate spatial arrangement.

\section{Exploration of Matrix Configuration}

Matrix configuration was the configuration most difficult to be explored in the presence of a cerebellar damage. Total errors, search efficiency, no visits, and perseverative errors reached the worst values in HCbed animals, indication of the challenge the matrix represented. In the absence of structured search patterns along rows or columns, a reasonable strategy for exploring the Matrix configuration could be a compromise between a foraging behavior that promoted an extended search among rewarded trays (i.e., visiting trays farther away than the previously visited one) and a behavior that limited the information to be stored (i.e., visiting the nearest tray). While control rats explored the matrix by alternating these two opposite patterns, HCbed rats did not succeed either in following a close directional principle or in varying between patterns. Their procedural impairment disarranged the exploratory strategies, and their performances collapsed. A specific feature of the matrix configuration was the presence of a central tray. While the control rats visited this inner tray with the same frequency than the remaining eight trays, HCbed rats visited it significantly less, strong indication that their procedural impairment affected the central exploration $[1,6]$. 


\section{Exploration of Cluster Configuration}

A search space organized in clusters or spatial chunks when explored in a principled manner provides the subjects with the possibility of chunking it, first visiting the locations within the same cluster and then moving to another one. The chunking theory predicts that once the chunks have been retrieved the burden on memory should be lightened from the total number of places to be explored (in this case, nine) to the number of clusters constituting the search space (in this case, three). Thus, the hierarchical organization of memory afforded by chunking substantially reduces the working memory load and promotes higher level performances [37-39]. Nevertheless, only the exhaustive depletion of a cluster before moving on to the next one collapses errors. Conversely, chunking appears to be a strategy of relative strength in rats in general [39] and in HCbed rats in particular, as indicated by studying rats' performances as a function of the angular relationships among the arms of a radial maze [40]. In a radial maze with arms differently rewarded, rats typically enter arms in a less preferable set before having sampled all arms in a more preferable one [38]. Even in the cluster configuration of the present research, control rats did not deplete the whole cluster before moving to the next one and returned to clusters they had already visited more than once, visiting the alreadydepleted trays. This less than perfect clustering tendency was further worsened by the presence of the cerebellar lesion. In comparison to intact rats, HCbed animals displayed significantly defective values of total errors, searching efficiency, perseverative errors, and differences in visiting internal and external trays. Interestingly, while most errors performed by controls were across cluster, HCbed rats performed more within-cluster than acrosscluster errors. Furthermore, the compelling tendency to stay, already demonstrated in other paradigms in the presence of cerebellar damage [8], induced neglecting some trays, as indicated by the considerable occurrence of no visits in the cluster configuration. These findings indicated that HCbed rats found it more difficult to remember the trays visited within a cluster than to identify whole, not-yet-depleted clusters. Since the two competencies are based on different memory functions acting on distinct time courses, the difference observed in HCbed rats could reflect a more limited storage capacity in working memory. This observation completely fits with the involvement of cerebellar circuits in the spatial working memory, already demonstrated in experimental $[3,41,42]$ and clinical $[43,44]$ studies.

The tendency of HCbed rats to visit (and revisit) the tray belonging to the same cluster can be also interpreted as a tendency to repeat the same behavior or as a difficulty to shifting from one setting to another or as a deficit in response inhibition, abilities demonstrated to be affected in cerebellar subjects $[23,45,46]$.

Trajectory Directness, Visit Correctness, Perseverations

In almost all configurations, all rats tended to reach a never-before-visited tray through a direct trajectory and to reach an already-visited tray through a tortuous pathway. The close correlation between trajectory direction and tray correctness is a sign that the rats reached a tray without hesitation when they were able to predict that the tray they were going to visit had not yet been depleted. Vice versa, uncertainty about the correctness of a visit was reflected in insecurity about the trajectory. Even if this correlation was basically maintained also in the presence of a cerebellar lesion, HCbed rats exhibited reduced percentages of it in all configurations, and at variance with controls they performed high percentages of direct trajectories to reach even already-visited trays. This latter pattern was displayed by HCbed rats especially in the matrix configuration, not by chance the configuration most difficult to be explored in the presence of cerebellar damage. The difficulties in changing strategy explain also the marked perseverative tendencies of HCbed animals in almost all spatial configurations. Perseverations are distinctive symptoms, characterized by prefrontal dysfunction, observed in human and experimental pathologies [47, 48]. They are also elicited by cerebellar damages [49]. This finding supports the view that cerebellar lesions might provoke "frontal-like" cognitive deficits and fits with clinical reports of severe problems in initiation/perseveration and in cognitive planning in cerebellar patients [47, 50-53]. The cerebellar engagement in the high-order processes falling under the umbrella term of executive functions, such as planning, sequencing, working memory, response inhibition, and cognitive flexibility, has been recently advanced $[46,53,54]$. In particular, the tendency to perseverate and the impossibility to inhibit patently wrong responses as well as the lack of flexibility in changing behavior might be due to impairment in planning intentional strategies, that is, in the ability to access and use different strategies effectively to change behavior in accordance with the context $[23,55]$.

In conclusion, the presence of a cerebellar damage loosened the capacity to make strategic use of the spatial structure of the search space.

\section{References}

1. Petrosini L, Molinari M, Dell'Anna ME (1996) Cerebellar contribution to spatial event processing: Morris water maze and T-maze. Eur J Neurosci 8:1882-1896 
2. Leggio MG, Neri P, Graziano A, Mandolesi L, Molinari M, Petrosini L (1999) Cerebellar contribution to spatial event processing: characterization of procedural learning. Exp Brain Res 127:1-11

3. Mandolesi L, Leggio MG, Graziano A, Neri P, Petrosini L (2001) Cerebellar contribution to spatial event processing: involvement in procedural and working memory components. Eur J Neurosci 14:2011-2022

4. Rondi-Reig L, Le Marec N, Caston J, Mariani J (2002) The role of climbing and parallel fibers inputs to cerebellar cortex in navigation. Behav Brain Res 132:11-18

5. Petrosini L, Leggio MG, Molinari M (1998) The cerebellum in the spatial problem solving: a co-star or a guest star? Progr Neurobiol 56:191-210

6. Mandolesi L, Leggio MG, Spirito F, Petrosini L (2003) Cerebellar contribution to spatial event processing: do spatial procedures contribute to formation of spatial declarative knowledge? Eur $\mathrm{J}$ Neurosci 18:2618-2626

7. Lalonde R, Botez MI, Boivin D (1987) Object exploration in staggerer mutant mice. Physiol Behav 41:115-117

8. Belzung C, Chapillon P, Lalonde R (2001) The effects of the lurcher mutation on object localization, T-maze discrimination, and radial arm maze tasks. Behav Genet 31:151-155

9. Lalonde R, Strazielle C (2003) The effects of cerebellar damage on maze learning in animals. Cerebellum 2:300-339

10. Lalonde R, Botez MI (1985) Exploration and habituation in nervous mutant mice. Behav Brain Res 17:83-86

11. Lalonde R (1987) Exploration and spatial learning in staggerer mutant mice. J Neurogenet 4:285-291

12. Lalonde R, Manseau M, Botez MI (1989) Exploration and habituation in Purkinje cell degeneration mutant mice. Brain Res 479:201-203

13. Lalonde R, Manseau M, Botez MI (1988) Spontaneous alternation and exploration in staggerer mutant mice. Behav Brain Res 27:273-276

14. Dahhaoui M, Caston J, Lannou J, Avenel S (1992) Role of the cerebellum in habituation exploration behavior in the rat. Physiol Behav 52:339-344

15. Joyal CC, Meyer C, Jacquart G, Mahler P, Caston J, Lalonde R (1996) Effects of midline and lateral cerebellar lesions on motor coordination and spatial orientation. Brain Res 739:1-11

16. Caston J, Chianale C, Delhaye-Bouchaud N, Mariani J (1998) Role of the cerebellum in exploration behavior. Brain Res 808:232-237

17. Foti F, Spirito F, Mandolesi L, Aversano M, Petrosini L (2007) Effects of spatial food distribution on search behavior in rats (Rattus norvegicus). J Comp Psychol 121:290-299

18. Molinari M, Petrosini L, Gremoli T (1990) Hemicerebellectomy and motor behaviour in rats. II. Effects of cerebellar lesion performed at different developmental stages. Exp Brain Res 82:483-492

19. Molinari M, Grammaldo LG, Petrosini L (1997) Cerebellar contribution to spatial event processing: right/left discrimination abilities in rats. Eur J Neurosci 9:1986-1992

20. Leggio MG, Molinari M, Neri P, Graziano A, Mandolesi L, Petrosini L (2000) Representation of actions in rats: the role of cerebellum in learning spatial performances by observation. Proc Natl Acad Sci U S A 97:2320-2325

21. Mandolesi L, Leggio MG, Spirito F, Federico F, Petrosini L (2007) Is the cerebellum involved in the visuo-locomotor associative learning? Behav Brain Res 184:47-56

22. Colombel C, Lalonde R, Caston J (2004) The effects of unilateral removal of the cerebellar hemispheres on spatial learning and memory in rats. Brain Res 1004:108-115

23. De Bartolo P, Mandolesi L, Federico F, Foti F, Cutuli D, Gelfo F et al (2009) Cerebellar involvement in cognitive flexibility. Neurobiol Learn Mem 92:310-317. doi:10.1016/j.nlm.2009.03.008
24. Mandolesi L, Foti F, Cutuli D, Laricchiuta D, Gelfo F, De Bartolo P, Petrosini L (2009) Features of sequential learning in hemicerebellectomized rats. J Neurosci Res. doi:10.1002/jnr.22220

25. De Lillo C, Visalberghi E, Aversano M (1997) The organization of exhaustive searches in a patchy space by capuchin monkeys (Cebus apella). J Comp Psychol 111:82-90

26. De Lillo C, Aversano M, Tuci E, Visalberghi E (1998) Spatial constraints and regulatory functions in monkeys' (Cebus apella) search. J Comp Psychol 112:353-362

27. Valsecchi P, Bartolomucci A, Aversano M, Visalberghi E (2000) Learning to cope with two different food distributions: the performance of house mice (Mus musculus). J Comp Psychol 114:272-280

28. Etienne AS, Maurer R, Séguinot V (1996) Path integration in mammals and its interaction with visual landmarks. J Exp Biol 199:201-209

29. Biegler R (2000) Possible uses of path integration in animal navigation. Anim Learn Behav 28:257-277

30. Parron C, Save E (2004) Evidence for entorhinal and parietal cortices involvement in path integration in the rat. Exp Brain Res 159:349-359

31. Buzsaki G (2005) Theta rhythm of navigation: link between path integration and landmark navigation, episodic and semantic memory. Hippocampus 15:827-840

32. Paz-Villagran V, Save E, Poucet B (2006) Spatial discrimination of visually similar environments by hippocampal place cells in the presence of remote recalibrating landmarks. Eur J Neurosci 23:187-195

33. Werner S, Bock O, Timmann D (2009) The effect of cerebellar cortical degeneration on adaptive plasticity and movement control. Exp Brain Res 193:189-196

34. Shaikh AG, Meng H, Angelaki DE (2004) Multiple reference frames for motion in the primate cerebellum. J Neurosci 24:4491-4497

35. Yakusheva TA, Shaikh AG, Green AM, Blazquez PM, Dickman JD, Angelaki DE (2007) Purkinje cells in posterior cerebellar vermis encode motion in an inertial reference frame. Neuron 54:973-985

36. Yakusheva T, Blazquez PM, Angelaki DE (2008) Frequencyselective coding of translation and tilt in macaque cerebellar nodulus and uvula. J Neurosci 28:9997-10009

37. Terrace HS, McGonigle BO (1994) Memory and representation of serial order by children, monkeys, and pigeons. Curr Dir Psychol Sci 3:180-189

38. Macuda T, Roberts WA (1995) Further evidence for hierarchical chunking in rat spatial memory. J Exp Psychol Anim Behav Proc 21:20-32

39. Cohen J, Pardy S, Solway H, Graham H (2003) Chunking versus foraging search patterns by rats in the hierarchically baited radial maze. Anim Cogn 6:93-104

40. Schenk F, Contant B, Grobety MC (1990) Angle and directionality affect rat's organization of visits, sequences and spatial learning in modular mazes. Learn Motiv 21:164-189

41. Martin LA, Escher T, Goldowitz D, Mittleman G (2004) A relationship between cerebellar Purkinje cells and spatial working memory demonstrated in a lurcher/chimera mouse model system. Genes Brain Behav 3:158-166

42. Caston J, Chianale C, Mariani J (2004) Spatial memory of heterozygous staggerer $(\operatorname{Rora}(+) / \operatorname{Rora}(\mathrm{sg}))$ versus normal $(\operatorname{Rora}(+) / \operatorname{Rora}(+))$ mice during aging. Behav Genet 34:319324

43. Geier CF, Garver KE, Luna B (2007) Circuitry underlying temporally extended spatial working memory. Neuroimage 35:904-915

44. Stoodley CJ, Schmahmann JD (2009) Functional topography in the human cerebellum: a meta-analysis of neuroimaging studies. Neuroimage 44:489-501 
45. Schmahmann JD, Sherman JC (1998) The cerebellar cognitive affective syndrome. Brain 121:561-579

46. Bellebaum C, Daum I (2007) Cerebellar involvement in executive control. Cerebellum 6:184-192

47. Hauser MD (1999) Perseveration, inhibition and the prefrontal cortex: a new look. Curr Opin Neurobiol 9:214-222

48. McDonald RJ, King AL, Foong N, Rizos Z, Hong NS (2008) Neurotoxic lesions of the medial prefrontal cortex or medial striatum impair multiple-location place learning in the water task: evidence for neural structures with complementary roles in behavioural flexibility. Exp Brain Res 187:419-427

49. Schmahmann JD (2004) Disorders of the cerebellum: ataxia, dysmetria of thought, and the cerebellar cognitive affective syndrome. J Neuropsychiatry Clin Neurosci 16:367-378

50. El-Awar M, Kish S, Oscar-Berman M, Robitaile Y, Schut L, Freedman M (1991) Selective delayed alternation deficits in dominantly inherited olivopontocerebellar atrophy. Brain Cogn $16: 121-129$

51. Grafman J, Litvan I, Massaquoi S, Stewart M, Sirigu A, Hallett M (1992) Cognitive planning deficit in patient with cerebellar atrophy. Neurology 42:1493-1496

52. Apollonio IM, Grafman J, Schwartz MS, Massaquoi S, Hallett M (1993) Memory in patients with cerebellar degeneration. Neurology 43:1536-1544

53. Schmahmann JD, Sherman JC (1997) Cerebellar cognitive affective syndrome. Int Rev Neurobiol 41:433-440

54. Bower JM (2002) The organization of cerebellar cortical circuitry revisited: implications for functions. Ann NY Acad Sci 978:135-155

55. Botez-Marquard T, Bard C, Léveillé J, Botez MI (2001) A severe frontal-parietal lobe syndrome following cerebellar damage. Eur J Neurol 8:347-353 